Bulletin d'Histoire Contemporaine de I'Espagne

$50 \mid 2016$

Les intellectuels en Espagne, de la dictature à la démocratie (1939-1986)

\title{
Au-delà des Pyrénées : les artistes catalans à Paris au tournant $\mathrm{du} \mathrm{XX}^{\mathrm{e}}$ siècle
}

\section{Laura Karp Lugo}

\section{(2) OpenEdition \\ Journals}

Édition électronique

URL : http://journals.openedition.org/bhce/905

DOI : 10.4000/bhce.905

ISSN : 1968-3723

Éditeur

Presses Universitaires de Provence

Édition imprimée

Date de publication : 1 décembre 2016

Pagination : 266-268

ISSN : 0987-4135

Référence électronique

Laura Karp Lugo, «Au-delà des Pyrénées : les artistes catalans à Paris au tournant du XXe siècle », Bulletin d'Histoire Contemporaine de l'Espagne [En ligne], 50 | 2016, mis en ligne le 09 octobre 2018 consulté le 24 septembre 2020. URL : http://journals.openedition.org/bhce/905 ; DOI : https://doi.org/ 10.4000/bhce.905 
Au-delà des Pyrénées: les artistes catalans à Paris au tournant du $\mathbf{X X}^{\mathrm{e}}$ siècle $^{1}$

\section{Laura KARP LUGO}

Attirés par l'émulation artistique et par les possibilités qu'offrait le marché de l'art français, les artistes catalans de la fin du $\mathrm{XIX}^{\mathrm{e}}$ siècle et du début $\mathrm{du} \mathrm{XX}^{\mathrm{e}}$ se sont rendus en masse à Paris, s'inscrivant dans un mouvement largement international. L'histoire que propose de considérer ce travail est non seulement celle des mobilités d'individus, des transferts artistiques et culturels, mais aussi celle des circulations, de la diffusion et de la réception d'œuvres. Quelle incidence eut l'origine de ces artistes dans leur parcours parisien? Quel fut leur degré d'acculturation? Quels réseaux parvinrent-ils à tisser? Quel fut l'accueil de leur œuvre, avec quelle lecture et quel usage? Comment s'élabora une identité à travers leur création? Grâce aux recherches motivées par ces interrogations, ce travail comble une lacune de l'historiographie de l'art catalan contemporain et des relations artistiques entre la Catalogne et la France, rejoignant les préoccupations actuelles sur les relations transnationales dans ce domaine.

Cherchant à contribuer à l'histoire culturelle et sociale des graveurs, peintres et illustrateurs issus du milieu artistique catalan du tournant $\mathrm{du} \mathrm{xx}^{e}$ siècle venus en masse tenter leur chance à Paris, cette

1 Thèse de doctorat d'Histoire de l'art, soutenue le 30 juin 2014, à l'INHA (Institut National d'Histoire de l'Art-université de Paris I-Panthéon-Sorbonne), sous la direction de $\mathrm{M}$. le Professeur Éric Darragon. Jury : Mmes. et MM. les prof. Éric Darragon, Paris 1 PanthéonSorbonne; Francesc Fontbona de Vallescar, Reial Acadèmia Catalana de Belles Arts de Sant Jordi, Barcelone; Rossella Froissart Pezone, Aix-Marseille Université; Bertrand Tillier, président, université de Bourgogne; Isabel Valverde Zaragoza, Universitat Pompeu Fabra, Barcelone. étude reconstitue un réseau mêlant des personnalités déjà largement étudiées (Hermen Anglada-Camarasa ou encore Ramon Casas et Santiago Rusiñol, chefs de file du modernisme), avec d'autres peu connues ou toutà fait oubliées (Josep Simont, dessinateur de L'Illustration de 1901 à 1921, et de 1933 à 1944, ou Eveli Torent, ami et collaborateur de Laurent Tailhade). Elle approfondit les questions autour de la création, de la diffusion et de la réception de ces artistes et de leurs œuvres. Le choix de la Catalogne s'explique par le statut de la région, que nourrissent son histoire, sa littérature, ses coutumes, sa langue. La reconsidération de la catalanité en tant qu'identité entraîne l'inclusion d'artistes nés dans d'autres provinces espagnoles dès lors qu'ils ont évolué artistiquement dans le milieu moderniste catalan. C'est le cas de Picasso, qui demeure à Paris étroitement lié à ses camarades tels Ricard Canals, Carles Casagemas, ou encore Ramon Pichot.

L'importance du contexte culturel, social, politique et économique conditionnant et justifiant le choix du sujet, un premier volet de ce travail s'attache à montrer d'une part la spécificité de la Catalogne par rapport au reste de l'Espagne (avec sa richesse industrielle et ses mouvements sociaux-politiques et culturels qu'ont été le modernisme et le noucentisme), et les réseaux des artistes barcelonais issus d'une même école (la Llotja), des mêmes académies privées, fréquentant les mêmes cercles artistiques, appartenant aux mêmes groupes d'amis, et empruntant les mêmes canaux de diffusion : presse et expositions. Le contexte catalan franchement francophile a donné aux artistes l'envie de voyager. Paris, qui diffusait son art depuis des décennies à travers la presse illustrée et les nombreuses expositions (les Expositions universelles mais aussi les Salons annuels), demeurait la destination de choix pour les Catalans - chez lesquels la distance avec Rome avait été prise bien plus tôt que dans 
le reste de la péninsule Ibérique. Ce constat se vérifie en analysant les prix et bourses de voyages attribués par le gouvernement espagnol et les différentes municipalités. La capitale française étant pour les Catalans la référence dans tous les domaines, de nombreux artistes ont fait le voyage sur une initiative privée et avec leurs propres moyens. C'est cet aspect qui ouvre le deuxième volet de cette étude. Les archives privées et publiques, les livrets des Salons et une abondante presse, tant catalane que française, permettent effectivement de dessiner la sociabilité parisienne de ces artistes: la reconstitution des réseaux est l'un des apports majeurs à cette histoire croisée. Au cœur des transferts culturels entre la Catalogne et la France, l'étude de deux revues qui n'avaient pas encore fait l'objet d'analyses fournit de nouvelles problématiques : sur le plan institutionnel en ce qui concerne Catalunya-Paris (la revue du Centre catalan de Paris); sur le plan privé pour L'Instant, revue franco-catalane d'art et littérature. L'attention portée aux rapports produits par la Préfecture dévoile des éléments inédits sur les surveillances policières auxquelles étaient soumis certains Catalans, notamment littéraires, et qui ont conditionné d'une manière ou d'une autre le séjour et l'expression artistique d'un groupe d'artistes catalans de Paris. L'œuvre sociale de Joaquim Sunyer s'explique par cette réalité. Au fil des pages, les pratiques artistiques des Catalans de Paris sont analysées et mises dans le contexte artistique et culturel parisien. Parce que c'est à Paris que les Catalans ont, pour la plupart, acquis les compétences techniques de l'estampe, un chapitre est consacré à la gravure sur métal, à la xylographie et à la lithographie. Ce travail accorde, de même, une place de choix à l'illustration, qu'elle soit pratiquée régulièrement ou ponctuellement. Extrêmement abondantes, les collaborations dans la presse rendent compte de la diffusion de l'œuvre des Catalans à Paris.

Les Salons parisiens et, dans une moindre mesure, ceux de province ont été l'autre grand canal de diffusion pour ces artistes. Une analyse d'ensemble des exposants catalans par Salon et des sujets présentés aboutissent à des conclusions significatives et déterminent la suite du propos: choix du Salon, stratégies d'envois, thématiques choisies selon la nature de la manifestation. La production, majoritairement folklorique, que les artistes catalans exposaient dans ces Salons suscite de nouvelles hypothèses stimulantes. Les achats officiels, effectués la plupart du temps dans les Salons, montrent l'intérêt de l'État français pour l'art de ces artistes en tant que communauté étrangère que l'on voulait voir représentée en France. En même temps, ces achats officiels rendent compte du succès des artistes catalans à Paris. Dans la sphère privée, marchands, galeristes, collectionneurs et mécènes ont joué un rôle tout aussi important, que ce travail s'attache à éclairer.

Étant donné que les artistes catalans de la fin du XIX ${ }^{e}$ siècle et du début du $\mathrm{XX}^{\circ}$ sont considérés dans leur ensemble comme les plus avant-gardistes en Espagne, et que le voyage à Paris était motivé par la volonté d'échapper au conservatisme de l'enseignement officiel espagnol et du goût du public, les orientations thématiques et stylistiques qu'ils empruntent semblent paradoxales au premier abord; pour mieux les appréhender, elles sont replacées dans leur contexte. Le fait que la plupart d'entre eux aient choisi de produire une œuvre vouée à se fondre dans la masse de sujets commerciaux rend évidente la motivation première du voyage à Paris : celle de construire une carrière grâce à un marché de l'art unique à l'époque et en pleine expansion. Ce constat essentiel détermine l'orientation du troisième volet de cette étude dédiée aux voies de diffusion et à la réception critique, dans l'intention 
d'expliquer et de contextualiser les choix artistiques adoptés. L'accent est mis sur le goût des Français pour l'Espagne, considérée comme gracieuse, sensuelle et colorée, et ne pouvant être représentée dans leur imaginaire que par son folklore. L'étude de la réception critique éclaire non seulement l'accueil reçu par ces artistes et leur œuvre, mais aussi la manière dont la France, à travers la critique, gérait le goût du public et donc inévitablement la production des artistes étrangers venus faire carrière sur son sol. Les conclusions rendent compte de la complexité et de l'ambiguïté des rapports: l'œuvre des Catalans est parfois considérée comme espagnole et parfois comme appartenant à un peuple avec des coutumes et des mœurs propres. Et puisque les critiques ne se préoccupaient souvent pas de distinguer les spécificités régionales, ce travail reconsidère les artistes du point de vue qui était alors adopté par la critique française. Des conclusions plus globales se dessinent touchant à la réception de l'art espagnol à Paris : ainsi, une partie importante de la critique s'attachait à ramener ces artistes désormais cosmopolites, ayant franchi les Pyrénées, à leurs frontières nationales, fatalement subjectives et fictives. Dès lors, cet ouvrage permet de mieux appréhender la manière dont la capitale internationale de l'art - qu'était Paris - a réagi à l'intégration des étrangers et a accueilli leur œuvre. De fait, le goût et la réception ont orienté la production des Catalans de Paris vers des sujets capables d'assouvir l'imaginaire des Français et de satisfaire l'idée qu'ils se faisaient de l'Espagne. Ces sujets régionaux ont été mis en rapport avec les paysages bretons, thématique exploitée par les artistes français, qu'ils aient été ou non originaires de la région. L'accueil bienveillant de l'iconographie folklorique en France se justifie aussi par une démarche stratégique de la critique qui consistait à accentuer les spécificités de chaque pays, en l'occurrence l'Espagne, afin de préserver une école nationale unique et originale pour son pays. Le fait que les Catalans aient choisi, pour la plupart, de répondre à cette demande explique l'absence de critiques significatives, notamment au moment de la montée xénophobe suite à l'accrochage du Salon d'Automne de 1912.

En permettant une compréhension plus juste de la place des artistes catalans audelà des Pyrénées, ce travail vient enrichir l'historiographie des rapports artistiques entre la Catalogne et la France. L'étude de l'adaptation des Catalans à Paris et de la réinterprétation de leur œuvre à cette époque charnière, marquée par l'essor des nationalismes, permet de repenser la manière dont Paris recevait et considérait l'Autre. Elle appelle également à relativiser l'ampleur de l'ascendant que la France exerçait sur les artistes catalans, qui finalement y voyaient surtout une chance pour réussir à vivre de leur art, et interroge le rapport centrepériphérie en cherchant à définir la place de Barcelone en regard de Paris.

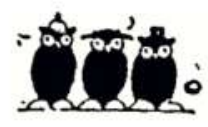

